\title{
Evaluation of a partial ring design for the INSERT SPECT/MRI system
}

\author{
Kell Erlandsson ${ }^{1 *}$, Debora Slavado ${ }^{1}$, Alexandre Bousse ${ }^{1}$, Brian Hutton ${ }^{1,2}$ \\ From PSMR 2015: 4th Conference on PET/MR and SPECT/MR \\ La Biodola, Isola d'Elba, Italy. 17-21 May 2015
}

'Institute of Nuclear Medicine, University College London, UK
The aim of the INSERT project is to develop a SPECT insert for a commercial MRI system, for performing simultaneous SPECT/MRI brain studies in humans. We have previously investigated various design options for the detector system, based on a complete ring of detectors. We are now considering a partial ring, due to space limitations. We have investigated the degradation in image quality with a partial ring as compared to a full ring, and the possibility of addressing the limitations by utilising MRI data during reconstruction. Noise-free data were generated by forward-projecting a cylindrical phantom with spherical inserts for a full-ring and a partial ring system, equipped with multislit- slat (MSS) and multi-pinhole (MPH) collimators. Poisson noise was added and images were reconstructed using ML-EM and MAP-EM with a smoothing prior and an anatomical prior. Contrast-recovery (CR) was calculated for the spheres in the lower part of the phantom compared to the top ones. Background $\mathrm{CoV}$ was also calculated. With noise-free data, CR was $77-84 \%$ for the MSS and $82-88 \%$ for the MPH partial-ring system with 400-1600 iterations. For noisy data and MAP-EM with a smoothing prior, CR was $78-80 \%$ and $81-82 \%$, and $\operatorname{CoV} 22-28 \%$ and $26-31 \%$, for the MSS and MPH systems, respectively. With the anatomical prior, CR was $85-89 \%$ and $87-91 \%$, respectively. With the partial ring-systems, the transaxial resolution in the lower part of the image is reduced. The degradation is slightly larger with MSS than MPH collimators, but the MSS collimator results in a lower noise-level. Some resolution can be recovered with more iterations, but the improvement is limited when regularisation is included. The anatomical prior offers both qualitative and quantitative improvement in image quality.

\section{Authors' details}

${ }^{1}$ Institute of Nuclear Medicine, University College London, UK. ${ }^{2}$ The Centre for Medical Radiation Physics at the University of Wollongong, Northfields Ave, Wollongong, Australia.

Published: 18 May 2015

doi:10.1186/2197-7364-2-S1-A47

Cite this article as: Erlandsson et al.: Evaluation of a partial ring design for the INSERT SPECT/MRI system. EJNMMI Physics 2015 2(Suppl 1):A47.

\section{SpringerOpen ${ }^{\odot}$}

(C) 2015 Erlandsson et al; licensee Springer. This is an Open Access article distributed under the terms of the Creative Commons Attribution License (http://creativecommons.org/licenses/by/4.0), which permits unrestricted use, distribution, and reproduction in any medium, provided the original work is properly cited. 